\title{
Complex needs survey: informing the development of a community recovery and rehabilitation team
}

\author{
Stavros Bekas ${ }^{1}$
}

The Psychiatrist (2013), 37, 192-197, doi: 10.1192/pb.bp.112.039958

${ }^{1}$ West London Mental Health NHS Trust, London

Correspondence to Stavros Bekas (stavros.bekas@nhs.net)

First received 6 May 2012, final revision 13 Dec 2012, accepted 15 Feb 2013

\begin{abstract}
Aims and method To inform the development of a new, recovery-oriented rehabilitation service, identify local needs and create a pathway for appropriate referrals, accelerating the build up of case-load. We surveyed 1353 secondary service users using a purpose-made needs assessment and case identification questionnaire, completed by nominated care coordinators.
\end{abstract}

Results Significant unmet need for rehabilitation and recovery-oriented interventions was found. The results were used to invite referrals, create a case-load of 150 and allocate the appropriate resources for the new team.

Clinical implications The results highlight the untapped recovery potential among users of mainstream secondary mental health services and support investment in rehabilitation at a time of resource restriction. This work also supports a similar, evidence-based approach to targeting appropriate referrals during the development and the early stages of rehabilitation services.

Declaration of interest None.
Redeployment of services in the National Health Service is often top-down, initiated by decision makers in response to a trend or politics, rather than as a result of local evidence gathering. With much of research and resources dedicated to symptom-focused interventions, psychiatric rehabilitation became unfashionable over the past decade as a kind of 'palliative care'. It lost significant resources and remains threatened in a climate of public finance retrenching. Recent focus on recovery meant a significant change of attitudes and models of care support rehabilitation once again. ${ }^{2}$ Whereas most old-style in-patient units are closing down, some services are reborn as community recovery and rehabilitation teams (CRRTs).

The population of the London borough of Tower Hamlets, currently at 220000 , grows rapidly and is characterised by wide ethnic, cultural and socioeconomic diversity, with large minority ethnic communities, especially Bangladeshi and Somali. The redeveloped, affluent parts of the borough neighbour some of the most deprived areas in the $\mathrm{UK}^{3}{ }^{3}$ Before the introduction of CRRTs, specialist rehabilitation was provided in a 13-bedded in-patient unit. Many service users with enduring illness stayed for lengthy periods on the acute wards or received residential care costing almost $£ 900000$ annually, with doubtful value for money. Stakeholders believed that a significant number could be discharged or moved to less supported accommodation more quickly with the provision of intensive community rehabilitation, making a case for a CRRT. Since pre-existing sources of data were inadequate, we designed this survey to identify rehabilitation needs, create a pathway for appropriate referrals and inform the development of a CRRT.

\section{Method}

\section{Participants}

We surveyed all 1353 service users living in Tower Hamlets, subject to care programme approach (CPA) under four locality community teams, an assertive outreach team and an early intervention service. From a database of active users we extracted names, date of birth, team, care coordinator and address, and applied the inclusion criteria. Recruitment was not required as users were not asked to provide information; respondents were their care coordinators. Managers decided that participation should be mandatory to maximise rate of response.

\section{Data collection}

Data were collected using a purpose-made questionnaire. After a literature review we identified a number of wellestablished instruments with relevant properties. The Community Placement Questionnaire, ${ }^{4}$ designed to examine long-stay in-patients preparing for their rehabilitation back to the community, is a standardised, staff-completed tool that records demographics, ratings of social functioning, problem behaviour, disability, social contact, accommodation and day-care needs. The Health of the Nation Outcome Scales (HoNOS) ${ }^{5}$ was developed as a measure of mental health and social functioning. The last four subscales in 
particular are pertinent to a recovery-oriented service, as they refer to problems with relationships, daily living, living conditions, occupation and activities. The Camberwell Assessment of $\mathrm{Need}^{6}$ is a schedule for the assessment of needs in mental health services, designed to provide the total number of needs, met needs and unmet needs, and the clinical version allows staff to plan patients' care. The Social and Occupational Functioning Assessment Scale (SOFAS) ${ }^{7}$ was also examined: developed as a modified version of the Global Assessment of Functioning scale for the purposes of DSM-IV Axis V, SOFAS produces a single rating between 0 and 100 describing the service user's level of social, interpersonal and occupational functioning, while excluding the severity of symptomatology.

No instrument from those reviewed was considered sufficient and the option of using them all was ruled out as impractical. Even though their perspectives are not identical, there is considerable overlap between items and they have shown substantial associations. ${ }^{8}$ Accordingly, we developed a new questionnaire incorporating ideas and items from these instruments, deeming any sacrifice of psychometric properties acceptable. A pilot questionnaire was tested by several care coordinators for feedback and improvement. The questionnaire aimed to produce a comprehensive user profile and to be suitable for referral and initial assessment. The first part asked about basic demographics, current care input, professionals involved and contacts. The second part had sections for residential needs, education, occupation, social and leisure activities, and an 'illness profile' that included diagnoses, level of engagement and adherence, risk factors and physical disabilities and needs. The last part incorporated a newly devised socio-occupational functioning scale (score: max. 10, min. 1), an estimate of the level of basic daily activities and the number of unmet needs. Respondents were finally asked whether the user was considered suitable for referral to the CRRT. Box 1 provides an outline of the questionnaire.

\section{Procedure}

One questionnaire for each service user was pre-populated by software with available data. Collated in packs with instructions, they were distributed by hand and collected regularly. Response was reinforced with email reminders, visits and feedback to managers. Data were transcribed and analysed using EpiData version 3.0 on Windows XP (EpiData Association, www.epidata.dk). Validity checks, prompts, jumps and calculations in the transcription programme increased validity and efficiency. We transcribed every 20th record on another data-set $(n=50)$ and compared them with the main data-set for consistency, finding minimal discrepancies (18 in 4500 fields). Paper copies were stored securely and the data-set was kept on three password-protected back-ups.

We compared the returned questionnaires with the total distributed, looking for significant differences based on gender, age and name of team. We calculated descriptive statistics using proportions and distribution means, including missing data when $>5 \%$. We also identified service users 'in need of rehabilitation' using arbitrary cut-offs: socio-occupational functioning score $<5$, unmet need score $>12$ and those identified by care coordinators as

\section{Box 1 Questionnaire outline}

Part 1

Demographics

1 Current mental health professionals involved.

2 Current mental health services involved.

Part 2

3 Current residential placement.

4 Are the service user's accommodation needs met?

5 If unmet/overmet, what ideally would be required?

6 Is the service user housed in or out of the borough?

$7 \mathrm{a}$ Is there an identified carer?

$7 \mathrm{~b}$ Have the carer's needs been assessed?

8 a Does the service user have children under age 18 ?

$8 \mathrm{~b}$ If yes, are Social Services aware or involved?

$8 \mathrm{c}$ Do the children live with the service user?

$8 \mathrm{~d}$ Are the children on the 'at risk' register?

8 e If yes, please specify the category.

9 Formal qualifications.

10 Current occupation.

11 Time spent involved in employment/meaningful activities.

12 Date of last paid employment.

13 Category of last paid employment.

14 Have the service user's occupational needs been met?

$15 \mathrm{Is} \mathrm{there} \mathrm{a} \mathrm{documented} \mathrm{occupational} \mathrm{action} \mathrm{plan?}$

16 Current activities.

17 Are the service user's social/leisure needs met?

18 Has an action plan for the unmet needs been created?

19 Has the service user been assessed by a clinical psychologist?

20a Primary diagnosis.

$20 \mathrm{~b}$ Comorbidity.

21 Number of psychiatric admissions in the past 5 years?

22 Duration of longest admission in the past 5 years.

23 How many times has the patient been detained under the Mental Health Act?

24 Concordance with medication.

25 Relapse indicators recorded in medical notes.

26 Engagement with services.

27 Risk factors.

28 Risk assessment/risk management plan in medical notes.

29 Does the person have any physical illness or disability?

30 How much help does the person receive from friends or relatives for physical health?

31 How much help does the person receive from local services for physical health problems?

32 How much help does the person need from local services for physical health problems?

33 Has a physical health assessment been done in the past year?

Part 3

34 Social and occupational functioning assessment scale (score 1-10).

35 What support is currently being provided for activities of daily living?

36 To what degree do existing services meet the patient's needs?

37 Please indicate which services you feel the patient should be considered for? 
potential referrals. Subgroup data were used in 11 univariate comparisons with the population producing confidence intervals at $\alpha=0.5$. These three subgroups were not mutually exclusive; any of these four characteristics was deemed as a proxy 'threshold' for possible services being offered by the CRRT.

Questionnaires were screened by two staff members and compared against draft referral criteria, including all users considered for referral by their care coordinator. Users thus identified were reviewed at a second stage at our weekly multidisciplinary meeting and those confirmed to be suitable were invited for a referral.

\section{Results}

From 1353 distributed questionnaires, 982 were returned, including 48 for users not subject to CPA or deceased, giving a response rate of $72 \%$. Comparing available data between distributed and returned questionnaires, no statistical differences were found. Demographics reflected the local population; mean age was 42 years and the vast majority were White Christian or Bangladeshi Muslim. Up to $15 \%$ needed an interpreter and $27 \%$ preferred to speak a language other than English. The majority (83.3\%) lived in general housing, with a significant proportion receiving informal $(14 \%)$ or professional $(3.2 \%)$ care at home; $13 \%$ lived in supported accommodation. There were 84 individuals waiting for general housing and 65 waiting for supported housing.

The majority of the service users were care-coordinated by a nurse or a social worker and they saw their care coordinator less than fortnightly. Only $30 \%$ regularly met a second mental health professional, usually a social or support worker. The majority (67\%) were reviewed by a psychiatrist less than monthly. Community teams appeared to be the only service provider for almost $70 \%$ of users. For the rest, the most significant input came from non-statutory organisations and local authority and housing associations, a significant $8.7 \%$ seeing the latter on a weekly basis or more often. About $34 \%$ received care from at least one friend or relative but only $19 \%$ of these received a carer's needs assessment. Another $22.6 \%$ (35\% for females) had underage children, but $80 \%$ of the care coordinators did not indicate whether the children lived in the same home or whether Social Services were involved.

Educational achievement appeared on average to be low, but a significant $51 \%$ had some qualification or training. Nonetheless, $73 \%$ were unemployed, $37 \%$ for more than 4 years. For $52 \%$ of the users, unmet occupational needs were reported, but only $22 \%$ had a documented plan to address them. For social activity, the majority (62\%) met friends and family regularly and engaged in leisure activities (39\%). Fewer were involved with resource centres (14\%), participated in faith activities (10\%) or engaged in physical exercise (1\%), and $2.5 \%$ had no meaningful activity at all.

Table 1 gives an overview of psychiatric problems, adherence and engagement. Although $37 \%$ of users also received adequate help for physical health problems, $64 \%$ had not had a physical assessment during the past year. The most prevalent risk areas were vulnerability (32\%), self-neglect (29\%), substance misuse (27\%), self-harm $(22 \%)$ and violence $(22 \%)$.

Tables 2 and 3 show the distribution of the patients on each level of psychosocial disablement and the existing support for activities of daily living, Table 4 provides the level of unmet needs in various domains of psychosocial function. Finally, Table 5 compares 11 variables between the 4 'in-need-of-rehabilitation' subgroups. Despite significant overlap between the latter, the cut-offs used did not identify exactly the same patients.

\section{Discussion}

Our survey was a strategic, targeted, consumer- and recovery-focused tool for the development of a new service offering a holistic approach to patient care. The results confirmed that there were unmet needs for recoveryoriented input among users of mainstream secondary

\begin{tabular}{|lc|}
\hline Table 1 Morbidity and adherence $(n=934)$ & \\
\hline & \\
\hline Diagnosis & 69.8 \\
Schizophrenia ${ }^{a}$ & 12.8 \\
Bipolar disorder & 8.5 \\
Anxiety/depression & 9.0 \\
Others & 28.3 \\
Substance misuse & 3.1 \\
Personality disorder & 3.1 \\
Intellectual disability & 37.0 \\
Physical comorbidity & \\
\hline Admissions in past 5 years & 0.4 \\
>10 & 19.4 \\
7-10 & 9.3 \\
4-7 & 50.9 \\
1-3 & 31.8 \\
None & \\
\hline Adherence: medication/engagement & $36.3 / 25.3$ \\
Full - active & $21.1 / 31.7$ \\
Full - passive & $21.3 / 12.8$ \\
Need support & $13.4 / 20.2$ \\
Resistant, inconsistent & $3.0 / 2.7$ \\
Other & \\
\hline
\end{tabular}

a. Includes schizoaffective disorder.

\begin{tabular}{|c|c|}
\hline $\begin{array}{c}\text { Table } 2 \text { Level of social and occ } \\
(n=934)\end{array}$ & nctioning $^{a}$ \\
\hline 10, Superior & 2.9 \\
\hline 9, Good & 10.3 \\
\hline 8, Slight impairment & 9.8 \\
\hline 7, Some difficulty & 20.5 \\
\hline 6, Moderate difficulty & 19.8 \\
\hline 5, Serious impairment & 14.5 \\
\hline 4, Major impairment, several areas & 9.0 \\
\hline 3, Inability to function, all areas & 2.2 \\
\hline$<2$, Persistent inability, at risk & 2.4 \\
\hline
\end{tabular}


Table 3 Support provided for basic tasks $(n=934)$

\begin{tabular}{|c|c|c|c|c|c|}
\hline & \multicolumn{5}{|c|}{$\%$} \\
\hline & Daily & Twice per week & Weekly & $>$ Two weekly & No help \\
\hline Cooking/getting food & 21.5 & 2.0 & 4.3 & 1.2 & 63.5 \\
\hline Shopping & 14.3 & 3.5 & 7.1 & 1.3 & 64.8 \\
\hline Domestic cleaning & 16.2 & 3.4 & 6.9 & 2.3 & 62.3 \\
\hline Budgeting/money & 14.4 & 1.6 & 6.7 & 1.6 & 63.9 \\
\hline Self-hygiene & 14.1 & 1.7 & 3.8 & 2.2 & 68.8 \\
\hline Using transport & 11.5 & 1.6 & 2.6 & 1.8 & 71.5 \\
\hline
\end{tabular}

\begin{tabular}{|c|c|c|c|c|}
\hline & No need, \% & Fully met need, ${ }^{a} \%$ & Partially met need, ${ }^{a} \%$ & Unmet need, \% \\
\hline Personal hygiene & 64.0 & 24.5 & 8.5 & 3.0 \\
\hline Daily living & 45.0 & 22.9 & 24.6 & 7.3 \\
\hline Relapse prevention & 12.8 & 45.9 & 28.3 & 2.5 \\
\hline Accommodation & 58.0 & 29.6 & 9.6 & 2.9 \\
\hline Social isolation & 35.5 & 18.6 & 37.7 & 8.2 \\
\hline Money management & 52.5 & 22.4 & 20.4 & 4.7 \\
\hline Leisure activities & 32.2 & 19.8 & 35.1 & 13.0 \\
\hline Work, training & 39.6 & 16.2 & 29.5 & 14.7 \\
\hline
\end{tabular}

a. Due to services offered.

\begin{tabular}{|c|c|c|c|c|}
\hline & \multirow[b]{2}{*}{$\begin{array}{c}\text { Total population } \\
n=982\end{array}$} & \multicolumn{3}{|c|}{ Mean $(95 \% \mathrm{Cl})$} \\
\hline & & $\begin{array}{c}\text { Functioning }<5 \\
n=306\end{array}$ & $\begin{array}{c}\text { Needs score }>12 \\
n=167\end{array}$ & $\begin{array}{c}\text { Possible referral } \\
\qquad n=371\end{array}$ \\
\hline Mean age, years & 41.85 & $42.93(41.35-44.52)$ & $39.10(37.00-41.20)$ & 41.59 (40.19-42.99) \\
\hline Males, \% & 61.2 & $63.0 \quad(56.7-68.9)$ & $66.9 \quad(58.5-74.4)$ & $63.5 \quad(57.8-68.9)$ \\
\hline 1 contact/week or more, $\%$ & 28.0 & $32.8 \quad(32.8-46.2)$ & $23.1 \quad(20.4-52.4)$ & $23.6(15.9-34.6)$ \\
\hline Schizophrenia, ${ }^{\text {a } \%}$ & 69.8 & $66.4 \quad(60.2-72.1)$ & $66.9 \quad(58.5-74.4)$ & $67.0 \quad(61.4-72.2)$ \\
\hline Living independently, \% & 64.1 & $52.1 \quad(45.8-58.4)$ & $57.7 \quad(49.1-65.8)$ & $52.4 \quad(46.7-58.1)$ \\
\hline Unmet housing needs, \% & 19.4 & $25.6 \quad(20.5-31.5)$ & $40.0 \quad(32.0-48.6)$ & $26.7 \quad(22.0-32.1)$ \\
\hline Poor engagement, $\%$ & 20.2 & $33.6 \quad(27.9-39.8)$ & $31.5(24.2-40.0)$ & $24.7 \quad(20.0-29.9)$ \\
\hline Physical illness, \% & 37.0 & $47.9 \quad(39.5-57.9)$ & $36.9 \quad(27.0-50.5)$ & $38.9 \quad(32.3-57.9)$ \\
\hline Functioning, SOFAS score & 6.21 & $3.97(3.81-4.14)$ & $5.4(5.06-5.73)$ & $6.36 \quad(6.12-6.59)$ \\
\hline Needs score & 8.35 & $11.02(10.26-11.78)$ & $15.46(15.05-15.87)$ & $10.50(9.78-11.23)$ \\
\hline Possible referral, \% & 37.7 & $50.4 \quad(44.1-56.7)$ & $59.2 \quad(50.6-67.3)$ & 100 \\
\hline
\end{tabular}

SOFAS, Social and Occupational Functioning Assessment Scale. ${ }^{7}$

a. Includes schizoaffective disorder.

b. Resistant to suggestions, fails to keep appointments.

services and supported investment in a new rehabilitation service in an era of resource restriction. ${ }^{9}$ Severe mental illness can become a major hurdle to living life to the full and offering opportunities to patients is essential in keeping hope alive. Equally important to the treatment of symptoms is the access to accommodation, training, occupation, entertainment and, above all, positive social roles and identity ${ }^{10,11}$ Providers seek more efficient ways of delivering psychiatric rehabilitation, recognising that it still has an important role in promoting the individual's potential to maximise quality of life and sense of agency., ${ }^{1,9-11}$
Community recovery and rehabilitation teams can provide localised, personalised care that offers users choice and combats stigma. ${ }^{9}$ This entails multidisciplinary care coordination of users in the community; expert assessments; transition work between supported placements; dynamic monitoring of morbidity, ability and physical health; and consultation on recovery and management of treatment resistance. Addressing social needs is essential, including education, occupation and finances, with emphasis on social inclusion and empowerment. ${ }^{9-11}$ Community recovery and rehabilitation teams have not been extensively studied 
but incorporate an eclectic approach, extrapolating support from research on intensive case management and occupational rehabilitation. ${ }^{12-14}$

Reliably screening needs and potential for change is the first step in targeting users presenting a degree of treatment resistance and chronic reduction of functioning that affects their quality of life and impedes independent living. The characteristics and needs of this population in Tower Hamlets were often assumed or noted on an individual basis but this was the first time that collective evidence was produced to inform service redevelopment.

Sociocultural factors had to be taken into account, such as the burden of stigma in Muslim communities and care of females with dependent children. The majority of service users had a diagnosis of schizophrenia, with multifaceted needs considering medication, psychological interventions, occupational therapy and nursing. Significant comorbidity with substance misuse indicated a need for specialist input, albeit compared with other inner-London areas this was less pronounced. ${ }^{15}$

Specific socio-occupational needs were highlighted. The respondents reported significant need in housing, especially low-support, independent and floating-support housing. In addition, users had extremely low levels of paid employment, a restricted range of meaningful activities and low educational attainment. Whether this was primarily due to reduced functioning or limited access to opportunities and support is hard to conclude. Additionally, 'functioning' and 'unmet need' scores seemed to identify different aspects, such that the latter were often associated with inadequate housing but not necessarily disability.

This survey indicated that although on average available services provided for basic care, input may be inadequate to cover complex needs. Overall, there was low level of intervention from psychiatrists, psychologists and occupational therapists, whereas many users could have benefited from a more multidisciplinary approach. In addition, non-statutory providers appeared underused. Occupational placements could partly address the remarkable lack of employment and brokering access to other outlets of psychosocial activity was also strongly indicated. It was unclear whether physical health needs were adequately addressed and known health risks were not adequately monitored.

Three characteristics were identified as most pertinent to our referral and assessment criteria:

1 low level of social, occupational and self-care activity, 2 unmet needs for support and opportunities, and

3 potential for engagement and meaningful change.

The last proved difficult to ascertain from the survey. The team applied clinical judgement in identifying potential, which could only be confirmed after a period of care. Conversely, diagnosis, symptomatology or physical disability did not differentiate users considered likely to benefit from the CRRT.

The results informed the efficient allocation and streamlining of resources. A case-load for the CRRT of 150 was deemed realistic, requiring 10 full-time care coordinators. The high need for psychological and occupational input supported the recruitment of occupational therapists, qualified psychologists, interpreters and bilingual support workers, as well as two psychiatrists, a full-time consultant and a junior at advanced training or specialty doctor level.

The survey provided some additional, unintended insights. A $72 \%$ response rate in a 'mandatory' project raises questions about care coordinators' attitudes towards service research. Missing data and discrepant answers in some items were beyond that expected to be due by chance, raising doubts on the detail of knowledge that many care coordinators have of users in their care and might indicate negative attitudes towards some user needs (e.g. co-occurrence of 'no meaningful activities' with 'occupational needs met'). Finally, contemplated referrals were often inappropriate; only half of those with low psychosocial functioning were included, whereas the disability of those deemed suitable was at the population average, with needs often skewed by unsuitable housing. This finding highlighted an additional need for staff training in rehabilitation and recovery.

\section{Study limitations}

Sampling error was insignificant. Non-sampling errors, namely inaccurate answers, flaws of the questionnaire, respondent confusion or other human factors are hard to quantify, but likely to have occurred in random directions. Care coordinators might have exaggerated the disablement of users they considered suitable for transfer or they might have downplayed the needs of those that they preferred to keep. This might also have influenced the response rate, with some groups under-represented. Respondents may also have felt that their work was being scrutinised, subtly influencing responses. The research team were insiders with motivation to identify rehabilitation needs, but quantification and a standardised questionnaire limited spurious interpretations. Destined for local service appraisal and planning, one would need to be cautious before generalising the results to other populations or circumstances. The choice of respondents, justified for practicality and ethical concerns, admittedly omitted the patient perspective. Evidence shows that user-rated unmet need is among the best indicators of quality of life ${ }^{16}$ and that agreement between staff and users contributes in predicting treatment outcomes. ${ }^{17}$ Although promotion of quality of life is at the core of modern rehabilitation, quantifying it remains a major challenge that has not been adequately tackled by research. $^{18}$ Items addressing quality of life were incorporated in our questionnaire, but further qualitative work might be a good next step.

\section{Acknowledgements}

I thank the staff of Tower Hamlets CRRT for their valuable contribution, especially Clive Denton, Team Manager, Julian Edwards, Trainee Psychologist and, above all, Dr Chris Sage, Consultant Psychiatrist, who conceptualised, designed and led this study.

\section{About the author}

Stavros Bekas MSc MRCPsych, specialty registrar (ST6) in general psychiatry, West London Mental Health NHS Trust, London. 


\section{References}

1 Wykes T, Holloway F. Community rehabilitation: past failures and future prospects. Inter Rev Psychiatry 2000; 12: 197-205.

2 Care Services Improvement Partnership, Royal College of Psychiatrists, Social Care Institute for Excellence. A Common Purpose: Recovery in Future Mental Health Services (SCIE Position Paper 08). SCIE, 2007.

3 Tower Hamlets Council. Indices of Deprivation 2010. Research Briefing 2011-03. Tower Hamlets Council, 2011.

4 Clifford P, Charman A, Webb Y, Craig TJK, Cowan D. Planning for community care: the Community Placement Questionnaire. Br J Clin Psychol 1991; 30: 193-211.

5 Wing JK, Beevor AS, Curtis RH, Park SB, Hadden S, Burns A. Health of the Nation Outcome Scales (HoNOS). Research and development. Br J Psychiatry 1998; 172: 11-8.

6 Phelan M, Slade M, Thornicroft G, Dunn G, Holloway F, Wykes T, et al. The Camberwell Assessment of Need: the validity and reliability of an instrument to assess the needs of people with severe mental illness. $\mathrm{Br} J$ Psychiatry 1995; 167: 589-95.

7 Goldman HH, Skodol AE, Lave TR. Revising axis V for DSM-IV: a review of measures of social functioning. Am J Psychiatry 1992; 149: 1148-56.

8 Slade M, Beck A, Bindman J, Thornicroft G, Wright S. Routine clinical outcome measures for patients with severe mental illness: CANSAS and HoNOS. Br J Psychiatry 1999; 174: 404-8.

9 Wolfson P, Holloway F, Killaspy H (eds) Enabling Recovery for People with Complex Mental Health Needs: A Template for Rehabilitation Services (Faculty Report FR/RS/01). Royal College of Psychiatrists, 2009.
10 Roberts G, Davenport S, Holloway F, Tattan T (eds) Enabling Recovery: The Principles and Practice of Rehabilitation Psychiatry. Gaskell, 2006.

11 Deegan P. Recovering our sense of value after being labeled mentally ill. J Psychosoc Nurs Ment Health Serv 1993; 31: 7-11.

12 Hutchinson DS, Razzano L. Multifaceted perspectives on program evaluation for psychiatric rehabilitation services. Psychiatr Rehabil J 2005; 28: 207-8.

13 Burns T, Creed F, Fahy T, Thompson S, Tyrer P, White I. Intensive versus standard case management for severe psychotic illness: a randomised trial. UK700 Group. Lancet 1999; 353: 2185-9.

14 Crowther R, Marshall M, Bond G, Huxley P. Vocational rehabilitation for people with severe mental illness. Cochrane Database Syst Rev 2001; 2 CD003080.

15 Menezes PR, Johnson S, Thornicroft G, Marshall J, Prosser D Bebbington $\mathrm{P}$, et al. Drug and alcohol problems among individuals with severe mental illness in south London. Br J Psychiatry 1996; 168 612-9.

16 Slade M, Leese M, Cahill S, Thornicroft G, Kuipers E. Patient-rated mental health needs and quality of life improvement. Br J Psychiatry 2005; 187: 256-61.

17 Lasalvia A, Bonetto C, Tansella M, Stefani B, Ruggeri M. Does staffpatient agreement on needs for care predict a better mental health outcome? A 4-year follow-up in a community service. Psychol Med 2008; 38: 123-33.

18 UK700 Group. Predictors of quality of life in people with severe mental illness. Study methodology with baseline analysis in the UK700 trial. $B r$ J Psychiatry 1999; 175: 426-32. 